\title{
Modelo de interfaz cerebro-computador de bajo costo como medio para mejorar la calidad de vida de las personas de la tercera edad
}

\section{Low-cost brain-computer interface model as a means to improve the quality of life of the elderly}

\author{
Domingo Valdes-Guevara ${ }^{1}$, Juan Mendoza-Rodriguez ${ }^{1} \&$ María Yahaira Tejedor-Morales ${ }^{1 *}$ \\ ${ }^{1}$ Universidad Tecnológica de Panamá, C.R. de Coclé, Grupo de investigación SoftSolution Group.
}

\begin{abstract}
Resumen La tecnología informática promete un gran potencial para mejorar la calidad de vida y fomentar la independencia de los ancianos. Los escenarios tecnológicos actuales, como las soluciones para problemas humanos sobre inclusión laboral, entornos de trabajo y seguridad, nos han inspirado a escribir este artículo y lo tomamos como una oportunidad para comprender cómo una abstracción de un concepto puede materializarse en una realidad. Al mismo tiempo, mediante el desarrollo de un modelo de interfaz informática, tendremos la oportunidad de ayudar a las personas mayores en las tareas y actividades cotidianas. Este artículo expone cómo, a través del procesamiento de señales cerebrales, se pueden llevar a cabo acciones o tareas mediante el uso de algoritmos de traducción. Anteriormente, el uso de la mente para controlar objetos solo sería posible dentro de nuestra imaginación más no en la realidad. Actualmente se están llevando a cabo proyectos, estudios e investigaciones en vía de desarrollo que permiten marcar una gran diferencia a tal punto que ahora es posible ir más allá de nuestra imaginación; ir más allá de aquello que alguna vez creímos imposible. Se presenta una propuesta para el desarrollo de un entorno para mejorar la calidad de vida de las personas de la tercera edad basado en los sistemas de interfaz cerebro-computadora (Brain-Computer Interfase-BCI), de modo tal que estos puedan apoyar en la interacción diaria con su entorno. Se plantea parte del panorama global y regional en donde esta conceptualización se cristaliza en aplicaciones que proveen una mejor calidad de vida.
\end{abstract}

Palabras clave Interfaz cerebro-computadora (BCI), EMOTIV Insight, RedRat-X, calidad de vida, ambientes de inclusión.

\begin{abstract}
Information technology offers great potential to improve the quality of life and foster independence of the elderly. Current technological advances such as solutions for human problems on labor inclusion, work environments and security have inspired us to write this article and take the opportunity to understand how an abstraction of a concept can be materialized in a reality. At the same time, through the development of a computer interface model we will have the opportunity to assist the older people in everyday tasks and activities. This article presents how, through signal processing of brainwaves, actions or tasks can be carried out using translation algorithms. Previously, the use of the mind to control objects would only be possible within our imagination but not in reality. Currently projects, studies and research are being conductred in this area, that are making a great difference to the point that now it is possible to go beyond our imagination; beyond what we once thought impossible. This paper presents a proposal for the development of an environment to improve the quality of life of the elderly based on the brain computer interfaz-BCI systems so that they can support the daily interaction with their environment. It considers part of the global and regional panorama where this conceptualization is crystallized in applications that provide a better quality of life.
\end{abstract}

Keywords Brain-computer interface (BCI), EMOTIV Insight, RedRat-X, quality of life, inclusion environments.

* Corresponding Author: maria.tejedor@utp.ac.pa

\section{Introducción}

La sociedad está siendo testigo del rápido aumento en el número y la proporción de personas mayores. Según cifras de las Naciones Unidas, en el 2017 se calcula que hay 962 millones de personas con 60 años o más, es decir, un 13 por ciento de la población mundial. Este grupo de población tiene una tasa de crecimiento anual del 3 por ciento. Europa es la región con más personas pertenecientes a este grupo, aproximadamente un 25 por ciento. Ese grado de envejecimiento de la población también llegará a otras partes del mundo para el año 2050, con excepción de África. Para el 2030, se estima que serán 1,400 millones de personas de edad avanzada en el mundo [1].

El gráfico 1 nos muestra que la realidad de Panamá no es distinta a la realidad mundial. Para el año 2050 se estima que la población de adultos mayores (60 años o más) crecerá paulatinamente y representará el $24 \%$ de la población 
panameña. Para el 2006 la población de adultos mayores había aumentado en un $103 \%$ en comparación de años anteriores. Para el 1 de julio del 2016 había 72,815 personas mayores de 80 años y a consecuencia se estima que para el 2019 el fondo de pensiones y jubilaciones entrará en déficit [2], lo cual obliga a definir nuevas alternativas por las cuales optar a fin de garantizar su seguridad social y su calidad de vida.

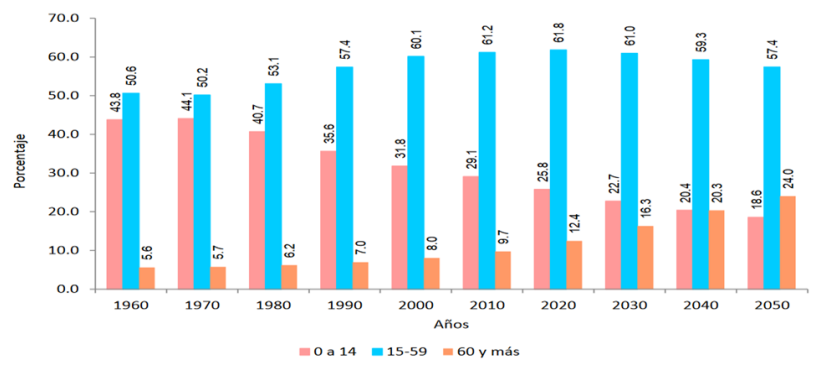

Gráfico 1. Distribución porcentual de las estimaciones y proyecciones de la población por grandes grupos de edad.

Fuente: Instituto Nacional de Estadística y Censo de Panamá.

Actualmente en Panamá la Ley 36 del 02 de agosto del 2016 establece las normativas a cumplir para la protección integral de los derechos de las personas adultas mayores. Sin embargo, solo el artículo 6 de esta ley hace referencia al derecho de vivienda de esta población, mas no establece la calidad del ambiente habitacional que las instituciones residenciales de protección de las personas adultas mayores deben poseer con el propósito de garantizar un entorno capaz de disminuir riesgos físicos, mantener la calidad de vida y generar espacios armónicos en el cual él y sus similares puedan formar parte de la sinergia de su entorno.

\section{Planteamiento del problema}

Factores como los altos números en la población de adultos mayores hacen suponer que no existe la disponibilidad suficiente de asilos para darles alojos al momento de llegar a su cúspide de edad. Actualmente en la ciudad capital de Panamá existe un promedio de 40 asilos para la población de adultos mayores. Algunos de estos asilos no se encuentran subsidiados. Independientemente de poseer o no algún tipo de subsidio para mantener operativos los programas para la tercera edad en cierta medida, la mayoría de ellos solo llega a cumplir con las condiciones básicas para la atención de esta población.

Países del continente europeo (Noruega, Suecia, Suiza, Alemania y Holanda) son un ejemplo a seguir por ser pioneros en implementar nuevas alternativas en busca de mejorar la calidad de vida de la población de adultos mayores como, por ejemplo, buenos servicios de salud y ambientes sociales favorables [3]. A pesar de esto, el progresivo aumento de la población de adultos mayores exige establecer nuevas alternativas capaces de garantizar el bienestar de este grupo de la población en sus años de adultez.
Teniendo en cuenta lo anterior y la creciente demanda por lugares habitacionales que brinden la satisfacción de poseer un ambiente capaz de velar por la salud, tranquilidad y seguridad de los adultos mayores, se requiere la generación de mecanismos que contribuyan a solventar esta necesidad.

Es ahí donde el uso de la tecnología puede ser visualizada como una alternativa viable que complemente y contribuya de manera significativa a este proceso. Por lo tanto, surge la oportunidad de presentar una propuesta para el desarrollo de una solución mediante el uso de tecnología BCI capaz de integrar factores internos (estado de ánimo), ideas, etc.) y externos (internet de las cosas) del entorno con la finalidad de proveer un ambiente controlado, seguro y satisfactorio.

\subsection{Comprendiendo el concepto de interfaz cerebro- computador}

La interfaz cerebro-computador es un medio que captura las ondas (señales) cerebrales y luego las procesa con la finalidad de traducirlas en órdenes o acciones que, exteriorizadas al medio ambiente, le permiten interactuar con su entorno. Es el proceso mediante el cual el pensamiento se convierte en acción.

\subsection{Desarrollo histórico}

La interfaz cerebro-computador no es algo relativamente nuevo, sino que paulatinamente ha ido evolucionando a través de los años al punto de lograr captar señales cerebrales mediante dispositivos no invasivos, como se muestra en la tabla 1.

Tabla 1. Evolución histórica de la interfaz cerebro-computador [4]

\begin{tabular}{|c|c|l|}
\hline Año & Investigador & \multicolumn{1}{|c|}{ Hechos relevantes } \\
\hline 1875 & Richard Caton & $\begin{array}{l}\text { Descubre la naturaleza eléctrica del } \\
\text { cerebro. Usó un galvanómetro para } \\
\text { observar impulsos eléctricos en la } \\
\text { superficie del cerebro vivo de algunos } \\
\text { animales. }\end{array}$ \\
\hline 1924 & Hans Berger & $\begin{array}{l}\text { Es el primero en registrar un EEG de } \\
\text { un cerebro humano y de presentar una } \\
\text { clasificación de los diferentes tipos de } \\
\text { ondas cerebrales. }\end{array}$ \\
\hline $1970-$ & $\begin{array}{l}\text { Universidad de } \\
\text { California, Los } \\
\text { Ángeles. }\end{array}$ & $\begin{array}{l}\text { Se inician las primeras investigaciones } \\
\text { para desarrollar dispositivos BCI con } \\
\text { fines médicos logrando desarrollar } \\
\text { prótesis neurales para rehabilitación. }\end{array}$ \\
\hline 2001 & varios & $\begin{array}{l}\text { Además de fines médicos, las } \\
\text { investigaciones se centran en la } \\
\text { industria de los videojuegos. Además, } \\
\text { se introducen en el mercado las } \\
\text { primeras interfaces no invasivas. }\end{array}$ \\
\hline
\end{tabular}

Para 1924 se logra establecer por primera vez las diferencias entre los distintos tipos de ondas cerebrales, las cuales se detallan a continuación en la tabla 2. Descubrimiento que marcó un gran cambio significativo en el futuro proceso de creación y desarrollo de los dispositivos BCI. 
Valdes (et al): Modelo de interfaz cerebro-computador de bajo costo como medio para mejorar la calidad de vida de las personas de la tercera edad

Tabla 2. Clasificación de las ondas cerebrales

\begin{tabular}{|c|c|c|c|}
\hline Nombre & Frecuencia & EEG & Descripción \\
\hline DELTA & 0,2 a $3,5 \mathrm{~Hz}$ & & $\begin{array}{lr}\text { Estado hipnótico, } \\
\text { hemisferio cerebral } \\
\text { derecho en } & \text { plena } \\
\text { actividad, } & \text { sueño } \\
\text { profundo, } & \\
\text { meditación. } & \\
\end{array}$ \\
\hline THETA & 3,5 a $7,5 \mathrm{~Hz}$ & & $\begin{array}{l}\text { Estado de vigilia, } \\
\text { equilibrio entre los } \\
\text { hemisferios } \\
\text { izquierdo y derecho, } \\
\text { plenitud, armonía. }\end{array}$ \\
\hline ALFA & 7,5 a $13 \mathrm{~Hz}$ & & $\begin{array}{l}\text { Relajación, } \\
\text { tranquilidad, } \\
\text { creatividad inicio de } \\
\text { actividad plena del } \\
\text { hemisferio izquierdo } \\
\text { y desconexión del } \\
\text { hemisferio derecho. }\end{array}$ \\
\hline BETA & 13 a $28 \mathrm{~Hz}$ & $N^{4}$ & $\begin{array}{l}\text { Estado de alerta } \\
\text { máxima, vigilante, } \\
\text { miedo, es la } \\
\text { situación normal } \\
\text { cuando estamos } \\
\text { despiertos, } \\
\text { conduciendo, o } \\
\text { trabajando en donde } \\
\text { estamos en estado de } \\
\text { alerta, ansiedad. }\end{array}$ \\
\hline GAMMA & $+\mathrm{de} 28 \mathrm{~Hz}$ & & $\begin{array}{l}\text { Estado de stress y } \\
\text { confusión. }\end{array}$ \\
\hline
\end{tabular}

\section{Metodología}

\subsection{Objetivos de la investigación}

El objetivo es utilizar una interfaz cerebro-computador (BCI) a través del cual el usuario podrá adaptar su ambiente/entorno a sus necesidades y confort para de ese modo mantener su calidad de vida acorde a las necesidades de su edad, como se muestra en la figura 1.

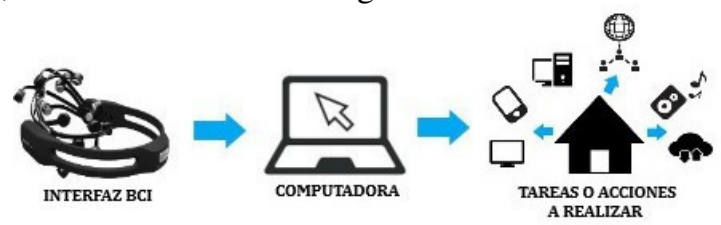

Figura 1. Mediante el uso del pensamiento se podrán realizar acciones, dar órdenes o realizar tareas.

Paralelamente, la interfaz monitoreará los estados de ánimos con el propósito de analizar y clasificar estos datos para identificar situaciones que puedan poner en riesgo la salud de los adultos mayores y, mediante el uso de un sistema de alertas, difundir un mensaje de advertencia al personal encargado del asilo para de ese modo darles la atención médica necesaria de forma inmediata.

\subsection{Materiales}

Como se muestra en la figura 2 , los materiales que se utilizarán para la obtención de las señales cerebrales y el control de los distintos tipos de dispositivos presentes en el entorno, serán:

\section{- Interfaz EMOTIV Insight}

- Dispositivo RedRat-X

- Software (desarrollado para la plataforma Windows 10 utilizando la colección de herramientas $\mathrm{BF}++$ ).

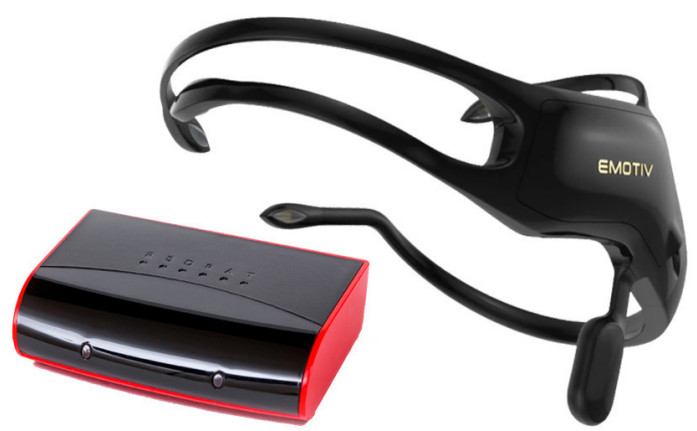

Figura 2. Hardware seleccionado para el desarrollo de la propuesta. RedRatX. EMOTIV Insight.

Tabla 3. Especificaciones referentes al modelo EMOTIV Insight

\begin{tabular}{|l|l|}
\hline \multicolumn{2}{|c|}{ Características } \\
\hline Sensor EEG & 5 canales \\
\hline Señales EEG & 128 muestras por segundo por canal \\
\hline Conectividad & Inalámbrica (PC y dispositivos móviles) \\
\hline Poder & Batería de 480mAh. Duración hasta 8 horas. \\
\hline Sensor de movimiento & 9 ejes \\
\hline Plataformas compatibles & Windows, MAC, iOS Android \\
\hline Costo & $\$ 299.99$ \\
\hline
\end{tabular}

Tabla 4. Especificaciones referentes al modelo RedRat-X Características Descripción

\begin{tabular}{|c|c|}
\hline Dimensiones & $11.5 \mathrm{~cm} \times 2.5 \mathrm{~cm} \times 7.5 \mathrm{~cm}$ \\
\hline Radio IR (parte frontal) & $0 \mathrm{~mA}$ a $250 \mathrm{~mA}$ en 100 pasos \\
\hline Conexión & Vía USB O Ethernet \\
\hline Indicadores LED & Brindan retroalimentación operativa \\
\hline Alimentación & $2 \mathrm{~W}$ \\
\hline 3 enchufes IR disponibles & $\begin{array}{l}\text { - Para usar con flashes IR } \\
\text { enchufables - 0mA a 100mA } \\
\text { en } 100 \text { pasos. } \\
\text { Para interactuar con sistemas } \\
\text { de distribución IR, como } \\
\text { Xantech, Buffalo, etc. } \\
\end{array}$ \\
\hline Costo & $\$ 334.50$ \\
\hline
\end{tabular}

Tabla 5. Especificaciones referentes al software a utilizar Características Descripción

\begin{tabular}{|c|l|} 
Windows 10 & $\begin{array}{l}\text { Es el último y vigente sistema operativo desarrollado } \\
\text { por Microsoft como parte de la familia de sistemas } \\
\text { operativos Windows NT. }\end{array}$ \\
\hline $\mathrm{BF}++$ & $\begin{array}{l}\text { Colección de herramientas de software y utilidades } \\
\text { desarrolladas para analizar, optimizar y diseñar Bio- } \\
\text { retroalimentación e interfaces Cerebro-computador }\end{array}$ \\
\hline
\end{tabular}




\subsection{Diseño}

Por definición, los ambientes inteligentes son espacios que hacen uso de las tecnologías de la información y comunicación, internet de las cosas y de los sistemas embebidos para crear entornos interactivos e inclusivos, reduciendo la brecha que existe entre el hombre y la tecnología al hacerla partícipe de las soluciones a los problemas cotidianos.

Se propone un escenario basado en el uso de tecnología BCI para mejorar la calidad de vida de las personas de la tercera edad. Dicho escenario tiene como principal actor al dispositivo EMOTIV Insight. Se escogió este modelo por su precio relativamente bajo y por tener disponible, tal como se aprecia en la figura 3, una aplicación móvil, mediante la cual se puede monitorear el estado mental del usuario y el correcto funcionamiento del dispositivo.

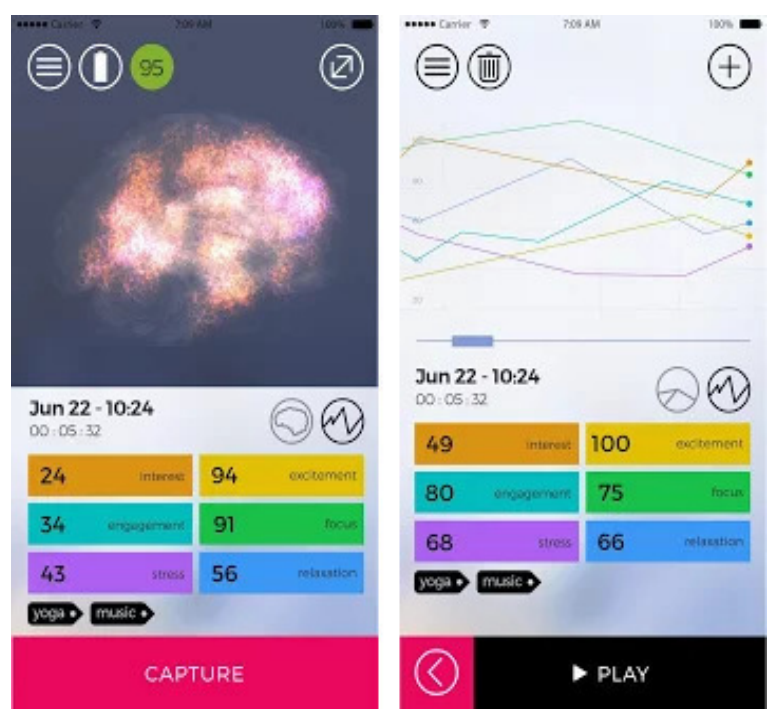

Figura 3. La aplicación MyEmotiv brindará información importante sobre el dispositivo y el estado mental del usuario.

Básicamente, es un electro encefalograma de cinco canales que capta señales de los lóbulos frontales (atención, toma de decisiones y planificación), lóbulos temporales (procesado auditivo, memoria, codificación y procesado del lenguaje) y los lóbulos parietal y occipital (integración sensorial y procesado de visión).

Este sistema tiene sus raíces en clínicas y hospitales, usados para estudiar condiciones médicas como lo son: coma, muerte cerebral, diagnosis de epilepsia y convulsiones y monitorear el sueño. El poder obtener una representación espacial del cerebro y lograr transformar pensamientos en acciones nos abre las puertas a una nueva forma de interactuar con el ambiente.

El dispositivo EMOTIV Insight será la interfaz por la cual se captarán las señales cerebrales del usuario que luego serán traducidas a binario mediante los algoritmos de traducción programados en el software que será desarrollado para sistemas operativos Windows 10 utilizando el paquete de licencia libre $\mathrm{BF}++$.

En este aspecto, el software podrá ser desarrollado optando por dos alternativas viables, como lo son:

- Captar los patrones exclusivos asociados al pensamiento que el cerebro produzca al momento de intentar realizar una acción, para luego darle una etiqueta exclusivamente a ese pensamiento. Como resultado cada vez que se tenga ese pensamiento provocará que esa acción ocurra, como se muestra en la figura 4.

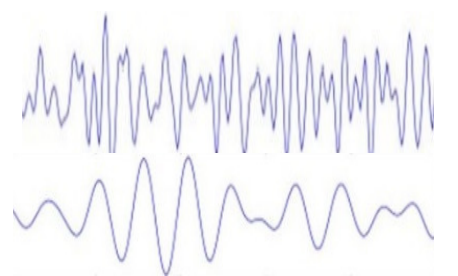

Figura 4. El cerebro produce distintos tipos de señales cerebrales de acuerdo al estado en que se encuentre.

- Como se aprecia en la figura 5, se pueden asociar las acciones a realizar con colores. Cada color representará una acción (encender/apagar dispositivos, abrir/cerrar ventanas, controlar sillas de ruedas, etc.) de modo que cada vez que el usuario desee realizar una acción solo deberá pensar en el color asociado a esa acción/tarea con la finalidad de llevar a cabo la ejecución de las instrucciones para la realización de esta.

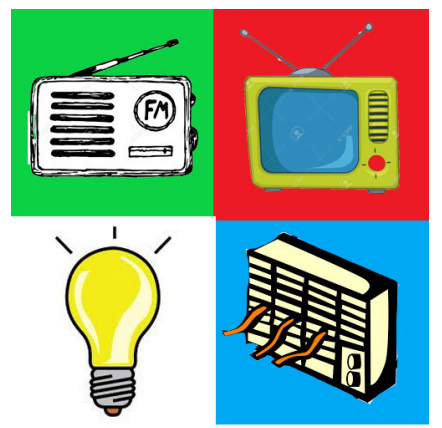

Figura 5. El uso de colores determinaría la acción a realizar dentro del entorno.

El aire acondicionado, el encendido/apagado de luces, televisores y cortinas eléctricas, son solo algunas de los dispositivos que podrán ser controladas mediante la interfaz cerebro-computador. Para este propósito se utilizará un dispositivo RedRat-X (control remoto por infrarrojos) que será conectado de forma sencilla al ordenador vía USB.

Una vez procesadas las ondas cerebrales mediante el uso de los algoritmos traductores programados, la señal será enviada a este dispositivo de emisión de señales infrarrojas a través del cual se distribuirán las señales que controlarán las acciones/tareas a realizar dentro del entorno.

Mediante la utilización de este emisor de señales vía infrarrojo se busca poder ajustar el entorno, de modo que se 
puedan satisfacer las necesidades del usuario, disminuyendo el desgaste físico sin que pierda participación en el proceso.

\section{Resultados}

Considerando el aumento progresivo que a futuro se registrará en la población de adultos mayores $\mathrm{y}$, por consecuencia, el aumento en la demanda de lugares que puedan satisfacer sus necesidades, brindar independencia y hacerlo activamente partícipe de su entorno, proponemos la creación de ambientes inteligentes controlados por una interfaz cerebrocomputador.

Su uso brindaría beneficios considerables a este grupo de la población panameña al ser capaz de mejorar considerablemente su calidad de vida. Abrirá las puertas a un nuevo capítulo, donde el desarrollo e innovación científica y tecnológica determinarán las pautas que trazarán grandes cambios en la sociedad panameña.

El modelo propuesto en este artículo haría uso principalmente de los siguientes elementos.

Tabla 6. Especificaciones referentes a los componentes del modelo

\begin{tabular}{|c|l|l|}
\hline Nombre & \multicolumn{1}{|c|}{ Descripción } & \multicolumn{2}{|c|}{ Función } \\
\hline EMOTIV Insight & $\begin{array}{l}\text { Casco de cinco } \\
\text { canales que captará } \\
\text { las señales } \\
\text { cerebrales. }\end{array}$ & $\begin{array}{l}\text { Monitorear el estado } \\
\text { cerebral para capturar las } \\
\text { señales cerebrales que se } \\
\text { generan al momento de } \\
\text { pensar en realizar una } \\
\text { acción. }\end{array}$ \\
\hline $\begin{array}{c}\text { Software } \\
\text { (Windows 10, } \\
\text { BF++) }\end{array}$ & $\begin{array}{l}\text { Software traductor } \\
\text { de pensamientos en } \\
\text { acciones. }\end{array}$ & $\begin{array}{l}\text { Procesar señales cerebrales } \\
\text { capturadas por el casco } \\
\text { EMOTIV Insight para } \\
\text { luego emitir las ordenes a } \\
\text { través del RedRat-X. }\end{array}$ \\
\hline RedRat-X & $\begin{array}{l}\text { Dispositivo emisor } \\
\text { de }\end{array}$ & $\begin{array}{l}\text { Emitir las órdenes de salida } \\
\text { conectarlas con los } \\
\text { infrarrojas. }\end{array}$ \\
& & $\begin{array}{l}\text { distintos tipos de } \\
\text { dispositivos que se } \\
\text { encuentran en el entorno. }\end{array}$ \\
\hline
\end{tabular}

Se escogió al dispositivo EMOTIV Insight por ser partícipe de muchas investigaciones previas, lo cual demuestra su gran eficiencia y eficacia. Por ejemplo, investigaciones han demostrado que el uso de las tecnologías cerebro-computador en el campo de la educación favorece la tarea del docente en su responsabilidad de ayudar a los alumnos en el proceso de enseñanza [5]. De igual manera, se ha logrado trazar nuevas estrategias para disminuir los niveles de estrés ocasionados por ciertas condiciones dentro del aula (ejemplo. borrar el tablero, arreglar sus bolsos, intervenciones verbales, etc.) en los estudiantes durante sus clases [6].

Dentro del campo de la medicina se han llevado a cabo investigaciones para desarrollar prótesis robóticas controladas por una interfaz cerebro-computador con la finalidad de otorgarle movilidad a personas que hayan perdido alguna de sus extremidades. Por otro lado, se han desarrollado sistemas de monitoreo remoto de bajo costo para señales biomédicas con la finalidad de registrar simultáneamente la actividad eléctrica cerebral y cardiaca. El sistema puede enviar datos biomédicos a una plataforma para análisis remotos [7].

Atendiendo a nuestro objetivo, cabe señalar que las tecnologías basadas en una interfaz cerebro-computador han abiertos las puertas al desarrollo de entornos laborales inclusivos con el propósito de integrar a personas discapacitadas al entorno social y laboral brindándoles la oportunidad de ser partícipes del entorno social evolutivo que constantemente se está llevando a cabo gracias al desarrollo e implementación de nuevas tecnologías.

Muchos son los beneficios que se podrán obtener gracias al uso de los sistemas BCI en el diseño y desarrollo de ambientes inteligentes para mejorar la calidad de vida para las personas de la tercera edad, como lo son:

- Provee independencia a las personas con problemas (motores) físicos.

- Otorga autonomía.

- Reduce costos de cuidados intensivos.

Lo interesante de los datos obtenidos mediante el uso del EMOTIV Insight radica en los beneficios alternos que se podrían obtener, como se muestra en la figura 6 . Los mismos no solo se limitan a los usuarios; se pueden diseñar vías de retroalimentación que brindarían información valiosa sobre la salud del usuario al personal encargado del recinto (asilo, hospital, etc.) y, gracias a la aplicación móvil, también a los familiares de ellos.

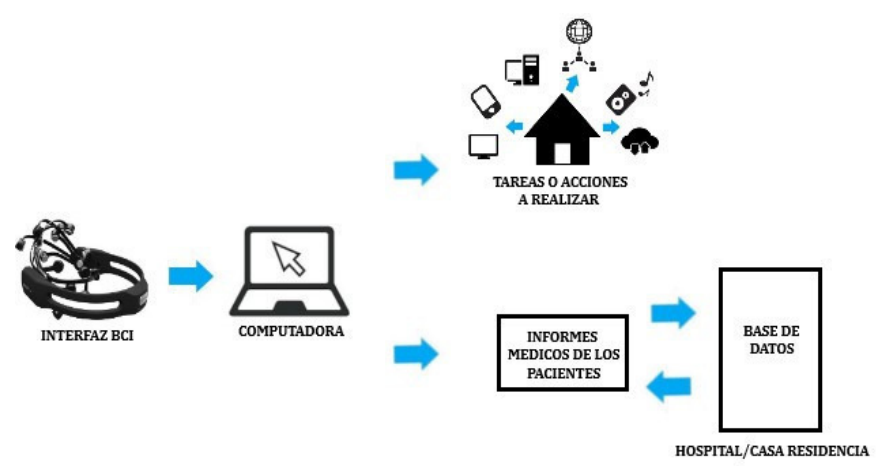

Figura 6. Feedback clínico. La tecnología BCI puede modificarse para registrar emociones o síntomas específicos para la medicina preventiva.

Al ser un sistema que se basa principalmente en la lectura de ondas cerebrales será posible observar, analizar y clasificar emociones, estados de ánimos, etc. al ser interpretadas se podrán monitorear niveles de estrés, irregularidad en las corrientes eléctricas cerebrales, Parkinson, demencia, terremotos cerebrales (epilepsia), etc., lo cual garantizará atención médica preventiva inmediata.

Finalmente, se propone el uso de colores para el desarrollo de la interfaz del software. Lo que se busca es evitar generar una sobrecarga mental por el exceso de actividad psicológica. 
$\mathrm{Al}$ hacer uso de colores como base que determinará las acciones a realizar, ayudamos a simplificar el proceso de pensamiento y a reducir la sobrecarga mental.

Atendiendo a lo anterior, debemos destacar que el uso de colores facilitará el uso de este sistema a los adultos mayores que padecen la enfermedad de Alzheimer, ya que, al utilizar colores, en este caso, cálidos y suaves estimularía positivamente al usuario al ofrecer, visualmente, un entorno relajante y tranquilizador.

Además, se ha demostrado que el uso de los colores es uno de los criterios utilizados por el cerebro para almacenar datos sobre objetos [8].

\section{Discusión}

Este entorno no está lejos de la realidad, sin embargo, necesita un estudio a profundidad que abarque más allá de las ventajas y beneficios que ofrece. Al igual que un estudio riguroso de problemas que podrían presentarse durante su puesta en práctica. Se pensó principalmente en satisfacer necesidades cotidianas, no obstante, a lo largo de la investigación ocurrieron una serie de ideas que podrían mejorar este entorno, creyendo fielmente en seguir mejorando este ambiente.

Cabe señalar que el entorno puede variar de acuerdo al tipo de necesidades que se desea satisfacer; el presente modelo ha sido pensado para disminuir riesgos en las personas de la tercera edad, pero el mismo puede ser rediseñado para crear ambientes de inclusión en el cual personas con discapacidades puedan sentirse plenamente activas y participes de las actividades laborales de la sociedad.

\section{Conclusiones}

La investigación realizada evidencia la importancia de las tecnologías basadas en BCI para promover la inclusión de personas con algún tipo de discapacidad en el entorno laboral, al ser capaz de crear ambientes y puestos de trabajos inteligentes, los cuales pueden ser controlados por el pensamiento y/o gestos a través de sensores vestibles en el cuerpo. Esto abre la puerta a nuevas investigaciones donde se puedan crear aplicaciones basadas en BCI que sean controladas no por el pensamiento sino por el estado de ánimo que posea la persona.

Por otra parte, su vital importancia reside en la capacidad de otorgarle segundas oportunidades a personas que hayan perdido algunas de sus extremidades debido a accidentes o por el contrario no posea, de nacimiento, ningún tipo de funciones motoras en las extremidades superiores. Más allá de otorgar oportunidades de inclusión laboral y de crear ambientes de trabajos adaptables, brinda nuevas alternativas de seguridad capaces de velar por la integridad del portador, al ser capaz de predecir a tiempo posibles accidentes donde su factor de origen sea el estado actual de las emociones del usuario.

Finalmente, Panamá cumple con las condiciones propicias para el desarrollo e implementación de tecnologías basadas en
BCI, como, por ejemplo, el de la Universidad Tecnológica de Panamá (Centro Regional Universitario de Chiriquí) en la cual propone la investigación y desarrollo de un prototipo para el control domótico basado en BCI para personas con movilidad reducida.

A pesar de aún no dar ese gran salto en el desarrollo e implementación de tecnologías BCI, Panamá se ha estado involucrando lentamente en el estudio de enfermedades que aquejan al cerebro como es el caso del Brain Treatment Center que lleva a cabo tratamientos que ayuden a dar alivio a las personas con autismo. Tal vez no es algo significativo en este campo, pero es un gran inicio en busca del estado mental óptimo en espera de la llegada de estas tecnologías.

\section{REFERENCIAS}

[1] NACIONES UNIDAS (2015). "Envejecimiento" [Online]. Available: http://www.un.org/es/sections/issuesdepth/ageing/index.html [Accessed: Ab. 2018].

[2] N. Alvarado (2016, Aug). "Aumenta la población anciana en Panamá; cifras revelan futura bomba de tiempo." [Online]. Available: https://www.tvn-2.com/nacionales/poblacionancianos-panama-aumenta_0_4543045720.html [Accessed: Ab. 2018].

[3] BBC Mundo (2014, Oct). “En qué países es mejor ser anciano?” [Online]. Available: http://www.bbc.com/mundo/noticias/2014/10/140930_ranking_p aises_personas_mayores_en [Accessed: Ab. 2018].

[4] A. Valencia, B. Juarez, A. Cortés y E. Velazquez. Estudio del estado del arte para el desarrollo de un motor gráfico que utilice Interfaz Cerebro-Computadora BCI para el aprendizaje de las TI. Revista de Sistemas Computacionales y TIC's, vol. 2, pp. 5-11, 2016.

[5] S. Prada Montilla (2017, Jun). "La Neurotecnología Educativa. Claves del uso de la tecnología en el proceso de aprendizaje" [Online].

Available: http://digibug.ugr.es/bitstream/handle/10481/47144/6-26.pdf?sequence $=1 \&$ is Allowed $=y$ [Accessed: Jun, 2018]

[6] L.A. Moreno Cueva, C.A. Peña Cortés, M. Maestre Delgado, S.G. Caicedo Villamizar, A. Pardo García, "Registro de neuroseñales con una interfaz cerebro-computador para estimar el nivel estrés en un estudiante durante una clase," INGE CUC, vol. 13, pp. 95101, 2017.

[7] J.M. Morales, C. Díaz-Piedra, L.L. Di Stasi, P. Martínez-Cañada, S. Romero. Low-cost Remote Monitoring of Biomedical Signals. IWINAC 2015: Artificial Computation in Biology and Medicine. Lecture Notes in Computer Science, vol 9107, 2015

[8] "El cerebro utiliza los colores para almacenar datos sobre objetos" [Online]. Available: http://notialzheimer.blogspot.com/2012/06/la-experienciareciente-influye-en-las.html [Accessed: Jun, 2018].

[9] I. Moreno. "Soporte para personas con movilidad reducida, basado en BCI" [Online]. Available: www.revistas.utp.ac.pa/index.php/memoutp/article/download/12 64/1702 [Accessed: Feb, 2018]. 
[10] R. Brescané. "Influencia del color en los enfermos de Alzheimer" [Online]. Available: https://www.google.com/search?q=influencia+del+color+en+los + enfermos+de+alzheimer\&ie $=$ utf- $8 \& o e=u t f-8 \&$ client=firefox-bab [Accessed: Jun, 2018].

[11] D. Santana, M. Ramírez y F. Ostrosky-Solís. Novedades en tecnología de la rehabilitación Una revisión acerca de interfaz cerebro-computadora, Revista Neurol, vol. 39, pp. 447-450, 2004.

[12] L. Da Silva Sauer, L, Valero Aguayo, F. Velasco Álvarez y R. Ron Angevin. Variables psicológicas en el control de interfaces cerebro-computadora. Psicothema, vol. 23, pp. 745-751, 2011.

[13] J. Minguez. "Tecnología de interfaz cerebro-computador" [Online].

Available: http://webdiis.unizar.es/ jminguez/Sesion001_UJI.pdf [Accessed: Feb, 2018].6

[14] J. Gutiérrez Martínez, J. Cantillo Negrete, R. Cariño Escobar y D. Elías Viñas. Los sistemas de interfaz cerebro-computadora: una herramienta para apoyar la rehabilitación de pacientes con discapacidad motora. Tecnología en Salud, vol. 2, pp. 62-69, 2013.

[15] M. Montiel Sandoval, P. Santana Mancilla y A. Guerrero Ibáñez. Interfaz cerebro-computadora para disminuir situaciones de riesgo en un entorno de conducción vial. Avances en Interacciones Humano-Computadoras, vol. 1, pp. 74-76, 2016.

[16] H. Valencia Ruíz, P. Santana-Mancilla, J. Guerrero Ibáñez y J. Contreras-Castillo. "Desarrollo de un prototipo de control remoto a vehículo por medio de una interfaz cerebro computadora" [Online]. Available: https://www.pedrosantana.mx/papers/valencia_aniei2016.pdf [Accessed: Feb, 2018].
[17] C. Escolano, I. Iturrate, J. Antelis y J. Minguez. "Dispositivos robóticos de rehabilitación basados en interfaces cerebroordenador: sillas de ruedas y robots para teleoperación" [Online]. Available:

http://webdiis.unizar.es/ jminguez/articles/EscolanoCea2009.pd f [Accessed: Feb, 2018].

[18] J. Muñoz Cardona, C. Muñoz Cardona, y O. Henao Gallo. Diseño de una estación de trabajo para personas con discapacidad en miembros superiores usando una interfaz cerebro-computador. Tecno Lógicas, Edición Especial, pp. 55-66, 2013

[19] R. Encarnación Mosquera. "Diseño de experimento: Aproximación metodología para el desarrollo de una aplicación interactiva de realidad virtual inmersiva, para recrear la terapia espejo en la rehabilitación de pacientes amputados de miembros inferiores con dolor de miembro fantasma" [Online], Available: http://revistas.utp.ac.pa/index.php/memoutp/article/view/1499 [Accessed: Feb, 2018]. 